\title{
Telemedizin
}

\section{Ein Arzt behandelt unmittelbar}

\author{
Fans von Fernbehandlungen per Chat und App verkennen, wie wichtig \\ der persönliche Kontakt zwischen Arzt und Patienten ist. Er ist nicht von \\ ungefähr fest in der Berufsordnung verankert.
}

— „Fernbehandlung“ gibt es schon lange, und sie war in den meisten Anwendungsbereichen schon immer erlaubt. Das mag verwundern, gelangt die Vokabel doch aktuell in einem eher negativ getönten Kontext wieder in die Öffentlichkeit: bei telemedizinischen Anwendungen. Mit diesen können Befunde, Diagnosestellung, Empfehlungen und Beratungen auch große Distanzen überwinden.

Kritik daran wird schnell als „Innovationsbremse" bezeichnet, wo doch in Wirklichkeit der Patientenschutz gemeint ist. Dass dieser ein hohes Gut ist und keinesfalls umgangen werden darf, versteht sich von selbst.

Wenn also die ärztliche Berufsordnung in ihrem $\$ 7$ Abs. 4 davon spricht, dass Ärzte „unmittelbar“ zu behandeln haben, so schützt dies auch vor Behandlung ,ausschließlich über Print- oder Kommunikationsmedien“. Einen Menschen nie gesehen, geschweige denn untersucht oder im Sinne einer Anamnese unärztlich angesehen.

\section{Im Alltag geht's doch auch!} von der Ärzteschaft erwarten. befragt zu haben, ihm dann aber eine Diagnose aufzustecken und gar eine Behandlungsstrategie, also eine Therapie $\mathrm{zu}$ empfehlen und entsprechend $\mathrm{zu}$ beraten - das wurde schon immer als

Mit der Möglichkeit, große Datenmengen über Distanzen sehr akkurat zu versenden, kann heute jeder Pennäler mittels Smartphone Bilder seiner Hautveränderung zu einem Freund schicken und nach dessen Meinung fragen. Diese aus dem Alltag bekannte Option führt dazu, dass die Patienten dasselbe auch

Nun setzt aber eine dem ärztlichen Selbstverständnis entsprechende Vorgehensweise nach dem Schema von Anamnese, Untersuchung, Diagnose, Aufklärung und Therapie immer noch den persönlichen Kontakt voraus. Nichts anderes ist mit dem Wörtchen „unmittel-

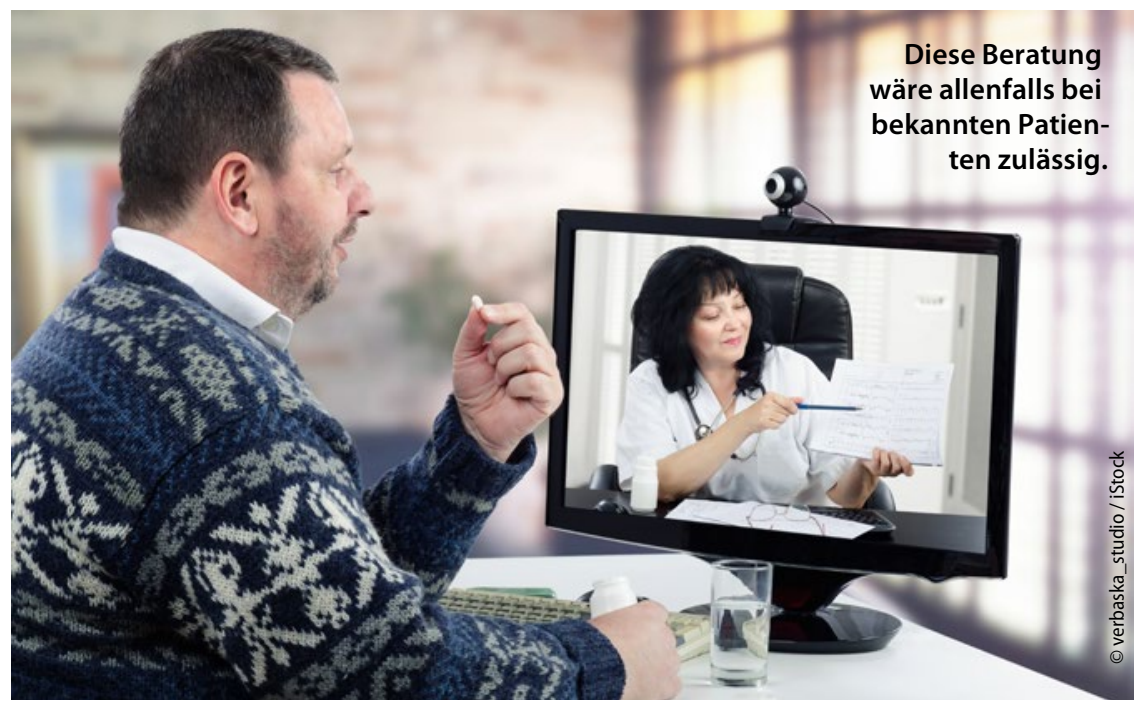

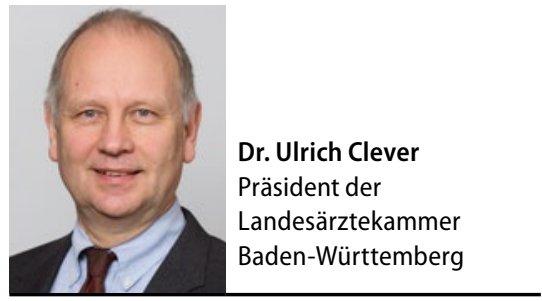

bar" gemeint, welches in der Berufsordnung einen so zentralen Platz einnimmt. Jeder soll eine umfängliche, seine Persönlichkeit umfassende Beratung durch den Arzt bekommen - und keine Parzialmeinung, wie sie etwa ein befreundeter Laie abgeben könnte.

Man bedenke auch: Patienten geben in Befragungen regelmäßig an, dass ihnen neben der konkreten medizinischen Hilfe beim Arztbesuch vor allem die Möglichkeit zum Gespräch wichtig ist.

\section{In Notsituationen per Telefon}

Selbstverständlich kommt es auch auf Angemessenheit an. Bei einer Augenverletzung etwa kommt es auf schnelle, effektive Hilfe an - und die kann in der Notsituation auch ein Arzt, der den Patienten nicht kennt, am Telefon oder Computer geben. Bei komplexeren Fällen, z. B. Problemen in Zusammenhang mit Diabetes mellitus, medikamentöser Antikoagulation oder neu auftretenden Allergien, reicht das bloße Foto, der bloße telefonische Bericht des aufgeregt Anrufenden nicht aus. Eine „unmittelbare“ ärztliche Inaugenscheinnahme erscheint dann auch dem Laien sogleich erforderlich. Selbst bei einer Fernberatung in Notsituationen sollte sie baldmöglichst nachgeholt werden.

Keine Bedenken bestehen gegen diese Art der Fernbehandlung hingegen, wenn der Patient sich bei seinem eigenen Hausoder Facharzt meldet, der ihn regelmäßig betreut. Dann nämlich liegen alle relevanten Informationen bereits vor, und die ärztliche Verantwortlichkeit des Vorgehens ist gegeben.

- Der Autor ist Präsident der Landesärztekammer Baden-Württemberg 